\title{
Development of A Smart Insole System for Gait and Performance Monitoring
}

\author{
Kaur LEEMETS ${ }^{1, a}$, Tõnis TERASMAA ${ }^{1, b}$, Paul JAAKSON $^{1, c}$, Alar KUME $^{1, d}$, \\ Tarmo TAMM $^{1, e,{ }^{*}}$
}

${ }^{1}$ Intelligent Materials and Systems Lab, Institute of Technology, University of Tartu, Nooruse 1, Tartu, 50411, Estonia

akaur.leemets@ut.ee, btonis.terasmaa@gmail.com, paul.jaakson@me.com, ${ }^{\mathrm{d}}$ alar.kume@ut.ee,

etarmo.tamm@ut.ee

${ }^{*}$ Corresponding author

Keywords: Podometry, Pedobarometry, Plantar pressure sensors, Capacitive sensors, Sensor insole.

\begin{abstract}
The present paper reports on the development of an autonomous, lightweight, and robust insole pressure sensor array system. The system measures plantar pressure on the go and channels the information to a mobile device but also stores it onboard, enabling post-measurement data analysis. The collected data can be used to analyze posture and gait but also to measure the performance of an athlete. The application is not limited to sports as abnormal plantar pressure distribution is of interest for several fields of medicine, among them the early detection of diabetic foot ulceration. The materials, methods, and approaches considered and tested during the development are discussed. The target system is aimed at high signal reproducibility for periodic measurements, high response frequency and long-term stability.
\end{abstract}

\section{Introduction}

Wearable technology is becoming an increasingly integral part of our everyday life, and both the demand and the supply of new systems and diverse gadgets is growing. In particular, the field of wearable sensors is gaining ground, systems that can provide real world data describing the actions of human body during everyday activities, whether at work, sport or leisure. While the driving force of entertainment must not be underestimated, such novel wearable sensor systems can provide valuable medical information helping diagnosis and rehabilitation. With both professional and amateur enthusiast athletes aiming for ever higher performance, detailed data characterizing the force/power output, cadence combined with heart-rate monitoring, distance and altitude readings provide invaluable aid for both athletes themselves and their coaches.

As people spend a considerable time on their feet, gait and posture can affect the overall health of humans considerably. As with most (orthopedic) problems, it is much easier to fix gait and posture problems when they are diagnosed already in their early developing stage; such problems become difficult or almost impossible to be remedied as they become fully developed. Perhaps the easiest way to measure and diagnose various problems with feet, legs, pelvis, and spine is the measurement of plantar pressure maps while standing, walking, and running. While different approaches have been proposed [1] for discovering and diagnosing such problems, the stationary sensory platform variety has been most extensively used and developed [2], and these are often readily available in main medical centers. These platforms have a dense matrix of pressure sensors; they are large enough to capture a few consequential steps and are relatively accurate if operated properly. A major drawback to stationary systems is the fact that they are (usually) limited to indoor confined spaces, which together with the limited size can change the natural gait during measurements.

For sports and recreational applications, such stationary systems are obviously of much lower value. In addition to direct medical application, modern pedobarometry also impacts areas such as footwear design [3], analyzing athletes' performance and minimizing risk of injuries [4], improving balance [5], 
but also for security and personal identification [6,7]. Another common goal for pressure distribution analysis is the design of customized insoles for recovery, therapeutics, lowering the risk of neuropathy, or just increased comfort $[3,8]$.

For recreational and sports performance applications, mobile systems are required. While initially such systems were bulky and with limited autonomy, recent progress in reducing the size and power consumption of electronic components as well as the development of low power high transfer-rate wireless communication protocols has finally enabled the development of reliable, truly autonomous systems. The applicability and performance of some wireless systems has already been studied in the scientific literature $[9,10]$, while some more recent promising designs [11] have not been investigated in in close detail yet.

The aim of the our effort was to design, construct and test a self-contained prototype sensor system that can monitor the plantar pressure mapping of both feet both integrally and differentially autonomously and at high frequency during several hours, without disturbing the studied person, e.g. affecting normal motion [10]. Naturally, the system has to be as light and thin as possible, while it must not be too elastic or soft.

While designing a pedobarometric insole system, one can choose between several types of sensors. These include but are not limited to capacitive, piezo-electric, (piezo)resistive, even optical varieties. Our preliminary selection based on sensitivity/stability and cost effectiveness focused on the resistive and capacitive varieties while novel ionic electroactive sensors (resistive in working principle) were also developed and tested. All of these systems can reach reasonable reproducibility (error $<5 \%$ ), sensitivity (signal to noise ratio $>25 \mathrm{~dB}$ ) in required pressure range $(5-75 \mathrm{~N} \cdot \mathrm{cm}-1)$, with acceptably low hysteresis. The main shortcomings of the selected systems were found to be temperature sensitivity (resistive), the influence of external parasitic capacitances and static charges (capacitive), and creep (ionic electroactive).

\section{Materials and Methods}

\section{System Design}

The electronics setup of our sensing system was constructed around a custom-designed flexible polyimide circuit board. Frequency is used as the measured signal, converted from the charging of a capacitor through a resistor by means of local Schmitt triggers at each of the 24 sensors. Such setup enables us to use both resistive sensing and capacitive sensing with rather minimal alterations to the circuitry. In case of resistive sensing, the capacitor is fixed, while the compressible conductive/resistive carbon nanotube-silicone foam generates the signal; alternatively, a constant resistance can be used with a variable elastic capacitor as the sensing element. While the resistive version could perform acceptably even with just a centralized electronics setup, the miniature size of the required components and the increase of accuracy obtained by the distributed placement in case of the capacitive working principle supported the choice of localized components. While developing several systems in parallel, the capacitive setup turned out to be most reliable and also most consistently prepared - an important aspect for potential industrial production. In order to conserve space and for increased clarity, only the above mentioned best performing capacitive setup will be described in detail below.

The principal architecture of the developed capacitive sensory system is described in Fig. 1.

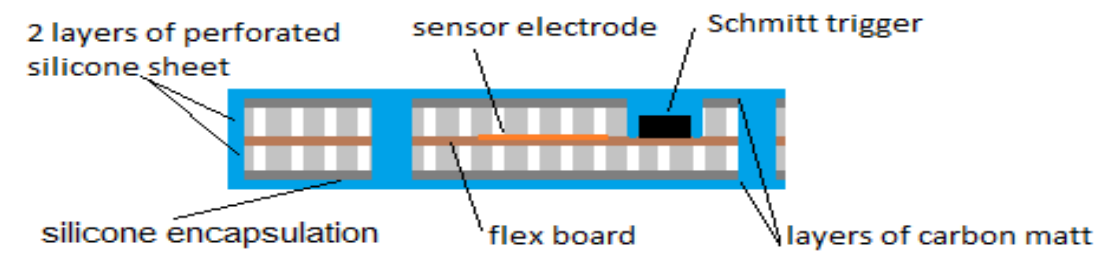

Fig. 1 Schematic representation of a sensing capacitive element of the insole 
The whole system is prepared as a laminate deign with the central flexible board containing the working electrodes of the sensors. The auxiliary electrodes are grounded and made of soft and flexible nonwoven carbon fiber matt with thickness lower than $0.1 \mathrm{~mm}$. This readily available material $(\mathrm{R} \& \mathrm{G})$ is both light-weight $\left(8 \cdot \mathrm{g} \mathrm{m}^{-2}\right)$ and thin, but also highly conductive (sheet resistance below $10 \mathrm{ohms}$ square $^{-1}$ ). The compressible sensory material was chosen to be perforated silicone sheet (unbranded) with thickness of $0.5 \mathrm{~mm}$. The hexagonally placed laser-cut perforation provided the necessary characteristics like improved sensibility (compressibility at different loads) and reduced mechanical cross-talk of neighboring sensors. The latter was reduced even further by cutting circular lines into the silicone sheet around each sensor, these lines had a depth of about $2 / 3$ of the thickness of the silicone sheet. Two sensor layers were used instead of just one in order to reduce the effect or random disturbances, both mechanical and electrical. The actual layers described above of the capacitive setup are depicted in Fig. 2.

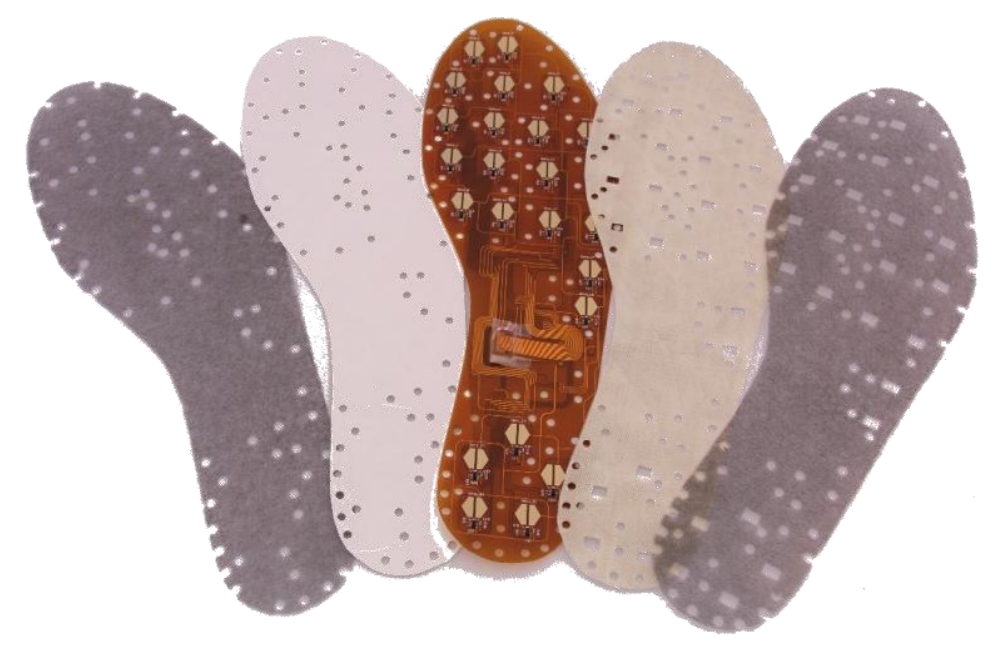

Fig. 2 Photograph of the layers used to form the sensory system before encapsulation. From left to right: bottom carbon fibre matt electrode, bottom silicone sheet, flexible electronics board, top silicone sheet and top carbon fibre matt electrode

The layers of different materials were placed in a 3D-printed mold of appropriate shoe-size and cast into silicone, providing integrity and encapsulation against the environment (mainly moisture). For increased adhesion and stability against delamination, all layers were perforated prior to casting. The laser-cut holes above sensor electrodes in the commercial silicone layer were covered with one-sided adhesive film in order to keep them unfilled.

The laminate design of the system should be well applicable for industrial production and can be modified easily to meet further design improvements, as required.

\section{Control Electronics}

Since dedicated Schmitt triggers were used next to each sensor, only frequency signal needs to be transported across the insole, this signal is much less affected by external parasitic capacitance/static charge effects than other types of signals would be. The 24 sensors were divided into 3 groups 8 sensors each. The groups were connected to a central microcontroller that reads frequency of the sensors. After performing calibration calculations, the data is sent either to the flash memory for storing and/or to a dedicated Bluetooth 4.0 LE transceiver unit that can transmit the data to a mobile device/computer. For different applications, either the differential (pressure mapping) or integral (overall force) of each step can be monitored. 3-axis accelerometers (in foot and heel) provide information whether the foot is moving or stationary while also providing some additional data about the orientation of force vectors connected to the pressure/force data measured. 


\section{Sensor Testing}

For dynamic testing of individual sensors in an insole, an automated hammer with adjustable frequency $(0.1-2.0 \mathrm{~Hz})$ and pressure $\left(1-350 \mathrm{~N} \cdot \mathrm{cm}^{-1}\right)$ was constructed. The force output from the hammer was calibrated using a Tedea-Huntleigh tension-pressure load-cell model 616. Static pressure measurements were conducted using a Gunt universal material tester model WP 300 in compression mode. For step analysis, a computer controlled piezo-electric dynamographic platform system (PD-3, Russia) was used.

\section{Results and Discussion}

\section{Individual Sensors}

An ideal sensor has a linear (or very well characterized nonlinear) response to the exerted force over the whole range of interest. According to the measurements with the automated hammer, the capacitive sensor system reached linearity with $95 \%$ of confidence level over the measured range. Other characteristics of paramount importance for sensory systems $[1,12]$ are the reproducibility, (long-term) stability and accuracy, often found lacking for proposed mobile systems. Our tested systems showed quick recovery (restored to $99 \%$ of initial response in less than $60 \mathrm{~s}$ ) from long term $\left(60 \mathrm{~min}, 30 \mathrm{~N} \cdot \mathrm{cm}^{-1}\right)$ constant pressure - simulating a person standing still. The creep was found to be below $1 \%$ during $60 \mathrm{~min}$ periodic force application at $1 \mathrm{~Hz}$.

\section{Integral Response}

In order to capture the sharp pressure changes in case of for example heel-strike running (

Fig. 3), a sampling rate of $50-100 \mathrm{~Hz}$ is required. While many stationary systems operate at $100-$ $200 \mathrm{~Hz}$, for portable systems the increased detail level is offset by the share amount of data generated which needs to be stored onboard or transmitted wirelessly. Our test systems operated at $50-75 \mathrm{~Hz}$.

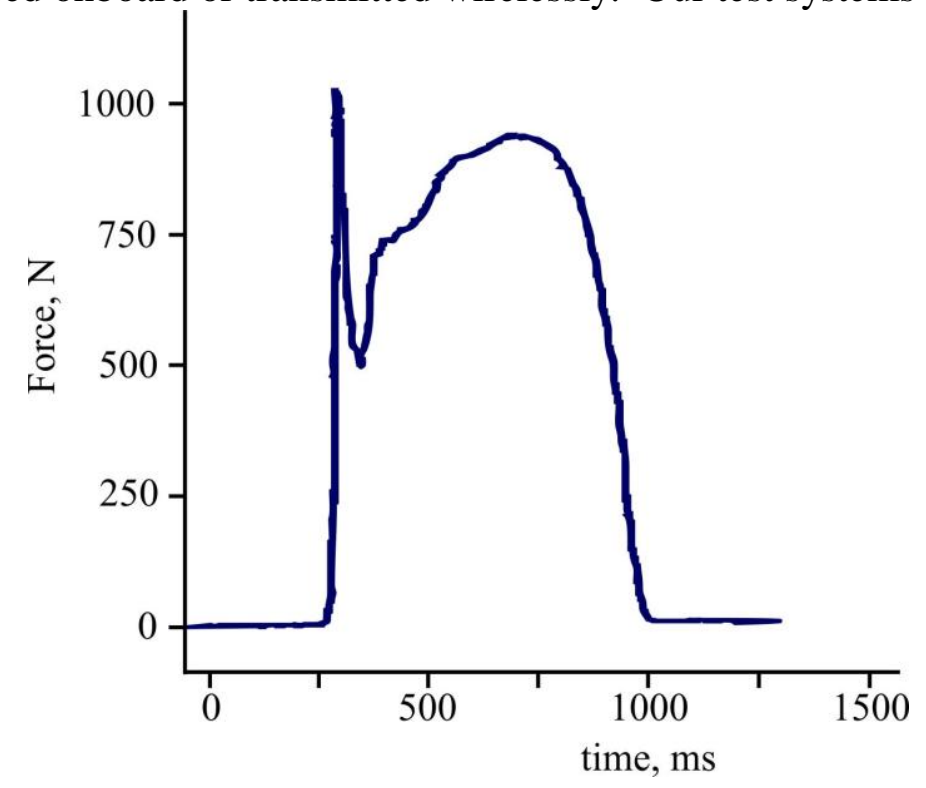

Fig. 3 Typical force response of a running step of middle-weight person

In order to capture the total force generated by the foot, a multi-step calibration was carried out on the dynamographic platform. While the forces detected by the platform and the insole showed inherent discrepancies in force timings for a counter-movement jump (Fig. 4), most likely caused by the elasticity of the insole itself and that of the footwear, the principal force dynamics of even such complex movements were found to be in good agreement. However, further testing and calibration schemes are required to provide consistent and reliable integral readings with physical force units for different conditions. 


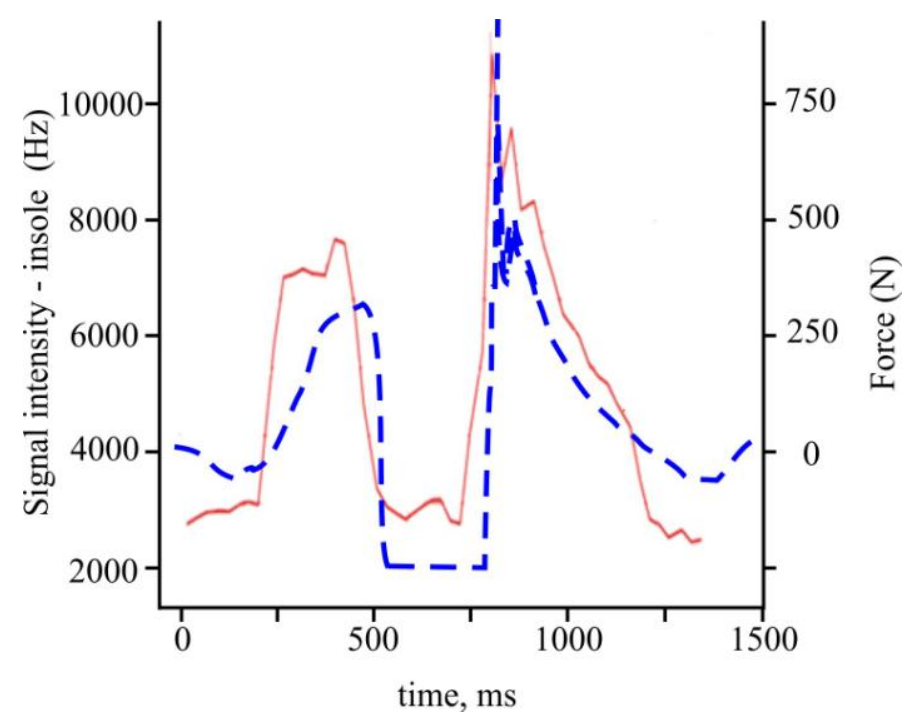

Fig. 4. Overlay of force response of insole (solid) and the respective output of tenso platform (dash) of a counter-movement jump

\section{Summary}

A thin, light weight and fully self-contained system for wireless pressure sensing insole has been developed that is applicable for both resistive and capacitive sensory materials. The described above capacitive version reached all target characteristics of signal quality, including accuracy, stability, reproducibility and linearity of response. Comparison against a dynamographic platform showed very good agreement of the integral force dynamics, however, further calibration is required before the system can be applied for obtaining physical force readings under diverse real-world conditions.

\section{Acknowledgement}

The project was supported by the SA Archimedes Materials Technology project SLOTI12166T. The assistance of specialists from the kinesiology group of University of Tartu and Sportlyzer Ltd. is gratefully acknowledged.

\section{References}

[1] Razak, A. H. A.; Zayegh, A.; Begg, R. K.; Wahab, Y.; Foot plantar pressure measurement system: A review. Sensors. 12 (2012) 9884-9912.

[2] Zammit G. V.; Menz H. B.; Munteanu S. E.; Reliability of the TekScan MatScan® system for the measurement of plantar forces and pressures during barefoot level walking in healthy Gait Analysis adults. J. Foot Ankle Res., 3 (2010) 11-20.

[3]Mueller, M. Application of plantar pressure assessment in footwear and insert design. J. Orthop. Sports Phys. Theor., 29 (1999) 747-755.

[4]Queen, R.; Haynes, B.; Hardaker, W.; Garrett, W. Forefoot loading during 3 athletic tasks. Am. J. Sports Med., 35 (2007) 630-636.

[5]Gioftsidou, A.; Malliou, P.; Pafis, G.; Beneka, A.; Godolias, G.; Maganaris, C. The effects of soccer training and timing of balance training on balance ability. Eur. J. Appl. Phys., 96 (2006) $659-664$.

[6]Yong, F.; Yunjian, G.; Quanjun, S. A Human Identification Method Based on Dynamic Plantar Pressure Distribution. In Proceeding of 2011 IEEE International Conference on Information and Automation (ICIA) (2011) 329-332. 
[7]Yamakawa, T.; Taniguchi, K.; Asari, K.; Kobashi, S.; Hata, Y. Biometric Personal Identification Based on Gait Pattern using Both Feet Pressure Change. In Proceeding of 2010 World Automation Congress (WAC) (2011) 1-6.

[8]Queen, R.M.; Abbey, A.N.; Wiegerinck, J.I.; Yoder, J.C.; Nunley, J.A. Effect of shoe type on plantar pressure: A gender comparison. Gait Posture, 31 (2010) 18-22.

[9]Grenez,F., Villarejo, M.V., Zapirain, B.G., Zorrilla, A.M., Wireless Prototype Based on Pressure and Bending Sensors for Measuring Gate Quality, Sensors (Basel) 13(11) (2013) 15861-15862.

[10]Bamberg, S.; Benbasat, A.Y.; Scarborough, D.M.; Krebs, D.E.; Paradiso, J.A. “ Gait analysis using a shoe-integrated wireless sensor system." IEEE Trans. Inf. Technol. Biomed., 12, 413-423 (2008).

[11]Information on: http://www.moticon.de/products/science-research\#science-research-sensor-insole.

[12] Low, D.C.; Dixon, S.J. Footscan pressure insoles: accuracy and reliability of force and pressure measurements in running. Gait \&Posture, 32(4) (2010) 664-666. 